\title{
Overexpression of long non-coding RNA CRNDE facilitates epithelial-mesenchymal transition and correlates with poor prognosis in intrahepatic cholangiocarcinoma
}

\author{
XIU-LIANG XIA, DONG XUE, TING-HAI XIANG, HUAI-YONG XU, \\ DE-KUN SONG, PEI-GUANG CHENG and JIAN-QIANG WANG \\ Department of General Surgery, The People's Hospital of Binzhou, Binzhou, Shandong 256610, P.R. China
}

Received May 25, 2017; Accepted September 20, 2017

DOI: $10.3892 / \mathrm{ol} .2018 .7815$

\begin{abstract}
The clinical significance and essential role of long non-coding RNA colorectal neoplasia differentially expressed (lncRNA CRNDE) have been well illuminated in various cancers. However, the function of CRNDE in intrahepatic cholangiocarcinoma (IHCC) has not been reported at present. The aim of the present study was to investigate the role of CRNDE in IHCC. Firstly, the relative expression of CRNDE was observed to be upregulated in IHCC cell lines and tissues. And high CRNDE expression was statistically associated with IHCC differentiation grade, lymph node metastasis, tumor-nodes-metastasis (TNM) stage and size. Survival analysis identified that high CRNDE expression is a predictor of worse overall survival (OS) and progression-free survival (PFS) in patients with IHCC. Moreover, high CRNDE expression was identified as an independent risk factor of IHCC poor OS and PFS. Further studies of in vitro assays suggested that CRNDE silencing could suppress the proliferation of HuCCT1 cells following CCK-8 and colony formation assays, while CRNDE ectopic expression in HCCC9810 cells promoted proliferation. Moreover, the migration and invasion of HuCCT1 cells were greatly repressed with CRNDE deficiency following Transwell and Matrigel assays. Accordingly, the motility of HCCC9810 cells was notably accelerated with CRNDE overexpression. Mechanistically, CRNDE was revealed to facilitate the epithelial-mesenchymal transition (EMT) of IHCC cells. In conclusion, these observations indicated that CRNDE could promote the clinical progression and metastasis of IHCC by facilitating EMT. CRNDE may be a novel prognostic marker and therapeutic target in IHCC.
\end{abstract}

Correspondence to: Professor Dong Xue, Department of General Surgery, The People's Hospital of Binzhou, 515 The 7th Huanghe Road, Binzhou, Shandong 256610, P.R. China

E-mail: xdbzsurgeon@163.com

Key words: intrahepatic cholangiocarcinoma, lncRNA CRNDE, EMT, metastasis, proliferation, prognosis

\section{Introduction}

Intrahepatic cholangiocarcinoma (IHCC) is the second most common primary hepatic malignancy. The incidence rate and cumulative mortality rate of IHCC have been steadily increasing during past decades $(1,2)$. Despite many advances having been made in the diagnosis and treatment strategies, IHCC is still a great challenge for doctors because of its heterogeneity and aggressiveness. Due to its insidious development process, the vast majority of IHCC patients are diagnosed at an advanced stage, with no access to radical surgical resection. Moreover, the recurrence rate of IHCC patients after surgical resection is high and advanced IHCCs are for the most part unresponsive to systemic chemotherapy and radiotherapy regimens (1). The current overall 5-year survival rate range from 15 to $40 \%$, and patients with unresectable IHCC will typically die within 12-24 months of diagnosis (3). Therefore, the molecular mechanisms of IHCC should be further elucidated, which may shed new light on identifying more effective diagnostic and prognostic markers and therapeutic targets.

Long non-coding RNAs (LncRNAs), defined as different types of RNA polymerase II-transcribed molecules with size greater than $200 \mathrm{nt}$ in length, have been discovered to regulate cellular biology at translational and posttranslational levels, the detailed mechanisms include recruiting transcription factors or chromatin-modifying complexes to their DNA targets, forming heterogeneous nuclear ribonucleoprotein complexes, acting as decoys to sequester RNA-binding proteins and microRNAs, or directly interacting with RNAs and DNAs by base pairing (4). Recent literatures have indicated that lncRNAs are involved in cancer initiation and progression, and numerous lncRNAs have been implicated to be deregulated in cancers and serve as essential regulators in many typical cancer biology pathways (5). Thus, lncRNAs-based therapeutics are considered as novel strategies in cancer treatment in the future (6).

LncRNA colorectal neoplasia differentially expressed (CRNDE), located on the long arm of chromosome 16 (16q12.2) of the human genome, was originally identified as a lncRNA in human colorectal cancer (7). The clinical significance of lncRNA CRNDE has been well studied in multiple tumors, such as colorectal cancer (7-9), renal cell carcinoma (10), and breast cancer (11). However, the potential role of CRNDE in 
Table I. Correlation between IncRNA CRNDE expression and IHCC clinicopathological characteristics.

\begin{tabular}{|c|c|c|c|c|c|c|c|}
\hline & \multirow[b]{2}{*}{ Parameters } & \multirow{2}{*}{$\begin{array}{c}\text { No. of } \\
\text { patients }\end{array}$} & \multirow[b]{2}{*}{$\begin{array}{l}\text { CRNDE } \\
\text { high/low }\end{array}$} & \multirow[b]{2}{*}{ P-value } \\
\hline \multirow{2}{*}{ Parameters } & \multirow{2}{*}{$\begin{array}{l}\text { No. of } \\
\text { patients }\end{array}$} & \multirow{2}{*}{$\begin{array}{l}\text { CRNDE } \\
\text { high/low }\end{array}$} & P-value & & & & \\
\hline & & & & TNM stage & & & 0.024 \\
\hline Age & & & 0.276 & $\mathrm{I}+\mathrm{II}$ & 58 & $19 / 39$ & \\
\hline$<60$ years & 58 & $28 / 30$ & & III+IV & 60 & $32 / 28$ & \\
\hline
\end{tabular}

Gender

Male

Female

80

$32 / 48$

TBIL

$$
\geq 20.4 \mu \mathrm{mol} / 1
$$

$<20.4 \mu \mathrm{mol} / 1$

AFP

$$
\geq 20 \mathrm{ng} / \mathrm{ml}
$$

$<20 \mathrm{ng} / \mathrm{ml}$

CEA

$\geq 5 \mu \mathrm{g} / 1$
$<5 \mu \mathrm{g} / 1$

CA19-9

$<100 \mathrm{U} / \mathrm{ml}$

$\geq 100 \mathrm{U} / \mathrm{ml}$

$27 / 27$

Cirrhosis

No

56

$27 / 29$

Yes

62

$24 / 38$

Satellitosis

Yes

No

Perineural invasion

$$
\text { Yes }
$$

No

Lymphovascular invasion

No

$19 / 23$

Yes

76

$32 / 44$

Grade

$$
\begin{aligned}
& \text { Well+moderate } \\
& \text { Poor+undifferentiation }
\end{aligned}
$$

Size

$$
<5 \mathrm{~cm}
$$

$\geq 5 \mathrm{~cm}$

T stage

$\mathrm{T} 1+\mathrm{T} 2$

$\mathrm{T} 3+\mathrm{T} 4$

$\mathrm{N}$ stage

$$
\text { No }
$$

N1

M stage
Table I. Continued.

\subsection{6}

CRNDE, colorectal neoplasia differentially expressed; IHCC, intrahepatic cholangiocarcinoma; TBIL, Total bilirubin; AFP, $\alpha$-fetoprotein; CEA, carcinoembryonic antigen; TNM, tumor-nodes-metastasis stages.

0.453

0.374

IHCC has not been investigated at present. This study aims to detect the expression level and clinical significance of CRNDE in IHCC, the potential mechanisms of CRNDE exerting its function were also investigated.
0.553

0.172

0.298

0.499

0.187

0.742

0.046

0.001

0.050

0.001

0.114

\section{Materials and methods}

Clinical samples collection. A total of 118 IHCC tissues and paired adjacent normal bile duct tissues were collected from primary IHCC patients who underwent surgery at the Department of General Surgery, the People's Hospital of Binzhou, Binzhou, Shandong Province, China. Informed consents were obtained from all the participants. No anti-cancer treatment was conducted for any patient prior to surgery. Obtained IHCC tissues and paired adjacent normal bile duct tissues were identified by pathological examination. This study protocol was approved by the Ethics Committee of the People's Hospital of Binzhou.

Cell culture and transfection. Normal human intrahepatic biliary epithelial cell (HIBEpic) and four human IHCC cells (HuCCT1, RBE, HCCC9810 and HUH28) were all purchased from the American Type Culture Collection. These cell lines were routinely cultured in DMEM (HIBEpic, HuCCT1, HUH28 and RBE) or RPMI-1640 (HCCC9810), supplemented with $10 \%$ fetal bovine serum (FBS) (Sigma, St. Louis, MO, USA), $100 \mathrm{U} / \mathrm{ml}$ penicillin sodium, and $100 \mathrm{mg} / \mathrm{ml}$ streptomycin sulfate. All cells were cultured in a humidified atmosphere containing $5 \% \mathrm{CO}_{2}$ at $37^{\circ} \mathrm{C}$.

HuCCT1 cells were transfected with siRNAs targeting CRNDE (siCRNDE-1 and siCRNDE-2) or a scrambled negative control (siNC) (GenePharma, Shanghai, China). HCCC9810 cells were transfected with CRNDE overexpressing vector (pcDNA3.1-CRNDE) or its control vector (pcDNA3.1-Vector) (GenePharma, China). The transfection was conducted with Lipofectamine 2000 (Invitrogen Life Technologies, Carlsbad, CA, USA) according to the manufacturer's protocol.

RNA extraction and RT-qPCR. RNA extraction from cell lines and clinical tissues was conducted with TRIzol reagent (Invitrogen, USA). To determine the mRNA expression level of target genes, SYBR Green fluorescent-based RT-qPCR was performed with an ABI PRISM 7900HT sequence detection system. Relative mRNA expression levels were calculated 
A

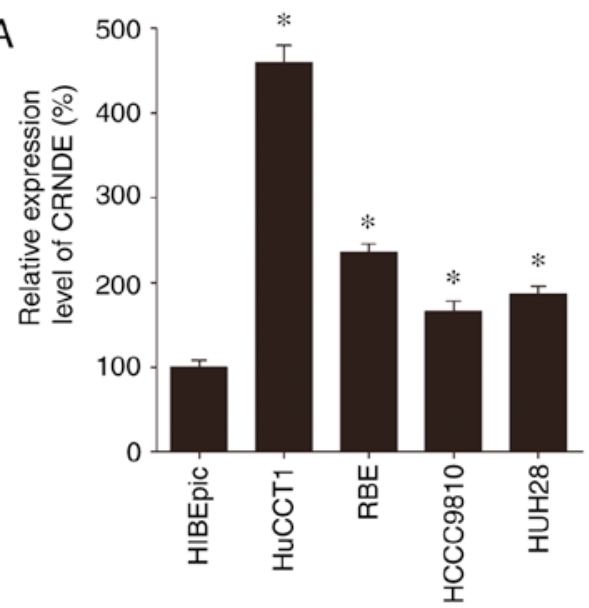

C
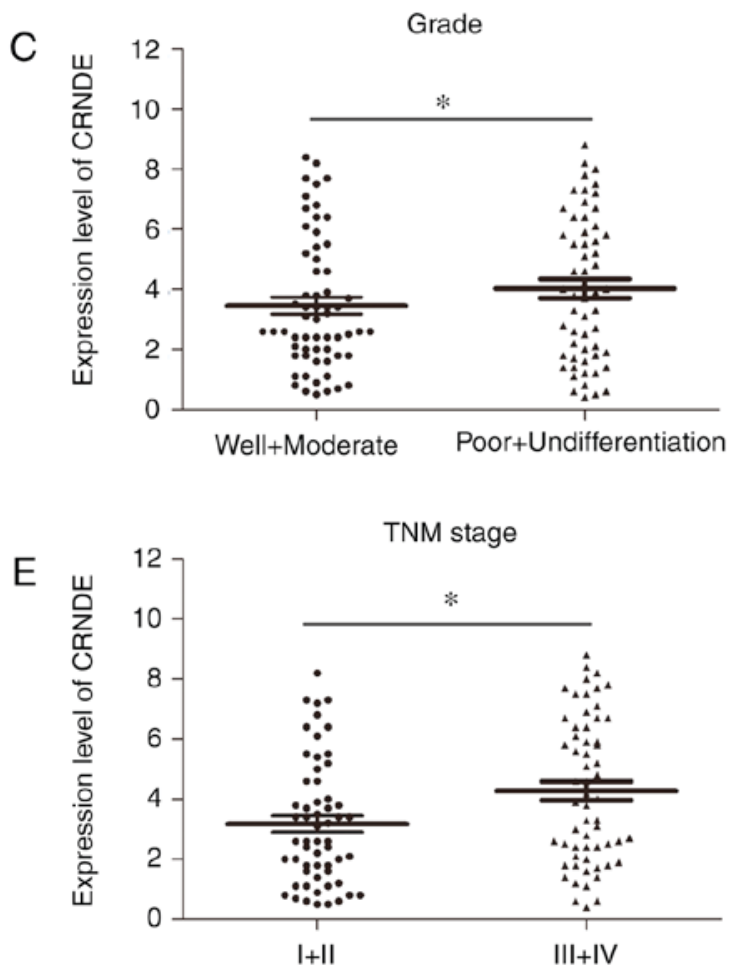

B

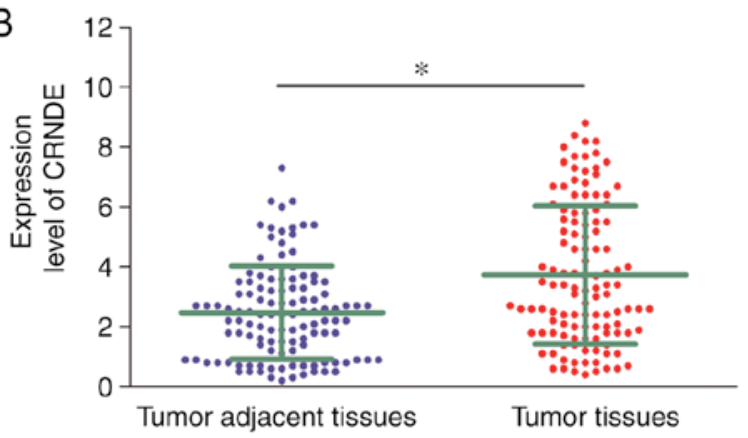

Lymph node metastasis
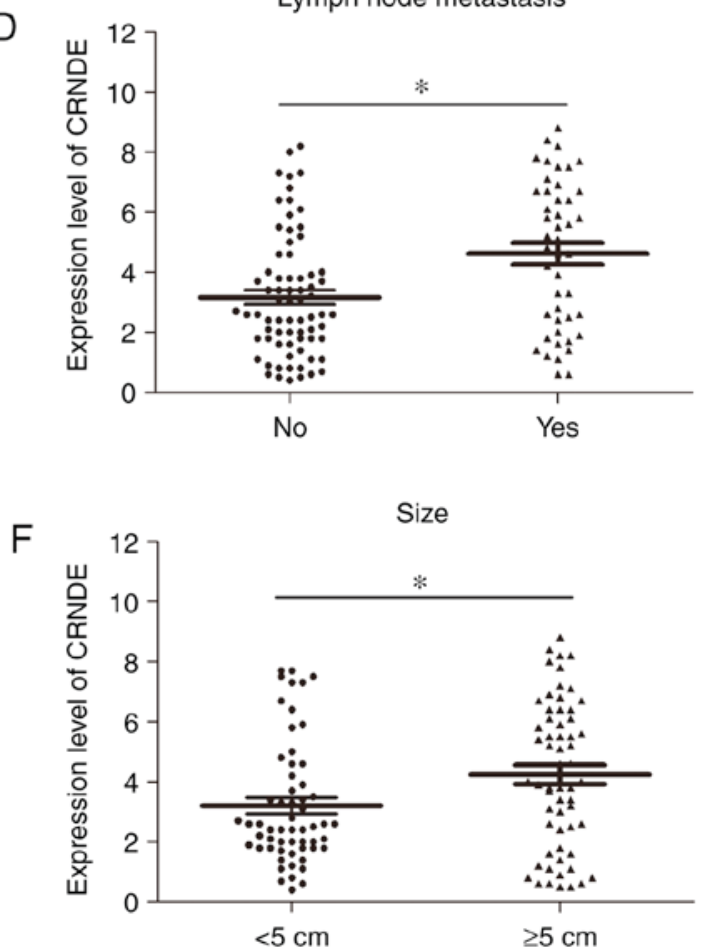

Figure 1. Up-regulation of CRNDE in IHCC correlates with clinical progression. (A) Expression of CRNDE was assessed in HIBEpic and four IHCC cell lines (HuCCT1, RBE, HCCC9810 and HUH28) by RT-qPCR. "P<0.05 vs. HIBEpic. (B) The expression level of CRNDE in IHCC tissues and paired adjacent tissues was analyzed by RT-qPCR assay. (C) Expression of CRNDE in well and moderate differentiated tissues compared with in poor differentiated and undifferentiated tissues on RT-qPCR assay. (D) Expression of CRNDE in tissues with lymph node metastasis relative to tissues without lymph node metastasis on RT-qPCR assay. (E) Expression of CRNDE in tissues with TNM stage I and II vs. tissues with TNM stage III and IV on RT-qPCR assay. (F) Expression of CRNDE in tissues with tumor diameter no less than $5 \mathrm{~cm}$ relative to tissues with tumor diameter less than $5 \mathrm{~cm}$ on RT-qPCR assay. "P $<0.05$ based on Student's t-test. Data are represented as Mean \pm SD. CRNDE, colorectal neoplasia differentially expressed; IHCC, intrahepatic cholangiocarcinoma; RT-qPCR, reverse transcription-quantitative polymerase chain reaction; TNM, tumor-nodes-metastasis stages.

by the $2^{-\triangle \Delta C T}$ method. GAPDH was used as internal control. Three independent experiments were conducted and each reaction was performed in triplicate. The primers were as follows: E-cadherin: 5'-CATTGCCACACATACACTCTC TTCT-3' (forward); 5'-CGGTTACCGTGATCAAAATCTC-3' (reverse); $\alpha$-catenin: 5'-GAGCTGTCTACGCAAGTCCC-3' (forward) and 5'-TTTCGGAGTACATGGGCAAT-3' (reverse). Vimentin: 5'-GGAACAGCATGTCCAAATCG-3' (forward); 5'-GCACCTGTCTCCGGTACTCA-3' (reverse). N-cadherin: 5'-GGTGGAGGAGAAGAAGACCAG-3' (forward) and 5'-GGCATCAGGCTCCACAGT-3' (reverse).
Cell proliferation assay. The Cell Counting Kit-8 (CCK-8) (Dojindo Molecular Technologies, Inc., Kumamoto, Japan) and colony formation assay were employed to determine the proliferation of HuCCT1 and HCCC9810 cells in accordance with the manufacturer's instructions. Briefly, HuCCT1 and HCCC 9810 cells were seeded into 96-well plates. After transfection, a total of $10 \mu \mathrm{l}$ CCK- 8 reagent was added to each well at designated time points $(24,48,72,96 \mathrm{~h})$. Then, cells were incubated for another $3 \mathrm{~h}$ followed by measuring the absorbance at $450 \mathrm{~nm}$ using an enzyme-linked immunosorbent assay plate reader. 
Table II. Univariate and multivariate analysis of clinicopathologic features for overall survival of IHCC patients.

\begin{tabular}{|c|c|c|c|c|c|c|}
\hline \multirow[b]{2}{*}{ Parameters } & \multicolumn{2}{|c|}{ Univariate analysis } & \multirow[b]{2}{*}{ P-value } & \multicolumn{2}{|c|}{ Multivariate analysis } & \multirow[b]{2}{*}{ P-value } \\
\hline & HR & $95 \% \mathrm{CI}$ & & HR & $95 \%$ CI & \\
\hline Age $\geq 60$ years vs. $<60$ years & 1.125 & $0.685-1.847$ & 0.643 & & & \\
\hline Gender female vs. male & 0.627 & $0.355-1.108$ & 0.108 & & & \\
\hline TBIL $\geq 20.4 \mu \mathrm{mol} / 1$ vs. $<20.4 \mu \mathrm{mol} / 1$ & 1.362 & $0.818-2.266$ & 0.235 & & & \\
\hline $\mathrm{AFP} \geq 20 \mathrm{ng} / \mathrm{ml}$ vs. $<20 \mathrm{ng} / \mathrm{ml}$ & 1.659 & $1.002-2.748$ & 0.049 & 1.193 & $0.682-2.086$ & 0.536 \\
\hline CEA $\geq 5 \mu \mathrm{g} / \mathrm{lvs} .<5 \mu \mathrm{g} / \mathrm{l}$ & 1.333 & $0.809-2.196$ & 0.259 & & & \\
\hline CA19-9 $<100 \mathrm{U} / \mathrm{ml}$ vs. $\geq 100 \mathrm{U} / \mathrm{ml}$ & 0.836 & $0.510-1.372$ & 0.479 & & & \\
\hline Cirrhosis yes vs. no & 1.102 & $0.672-1.808$ & 0.701 & & & \\
\hline Satellitosis yes vs. no & 1.369 & $0.799-2.346$ & 0.254 & & & \\
\hline Perineural invasion yes vs. no & 1.182 & $0.708-1.976$ & 0.522 & & & \\
\hline Lymphovascular invasion no vs. yes & 0.462 & $0.281-0.760$ & 0.002 & 0.530 & $0.313-1.224$ & 0.018 \\
\hline Grade poor+undifferentiated vs. well+moderate & 1.629 & $0.984-2.696$ & 0.058 & & & \\
\hline Size $\geq 5 \mathrm{~cm}$ vs. $<5 \mathrm{~cm}$ & 1.426 & $0.867-2.347$ & 0.162 & & & \\
\hline T stage (T3+T4) vs. $(\mathrm{T} 1+\mathrm{T} 2)$ & 1.444 & $1.030-2.024$ & 0.033 & 0.621 & $0.315-1.224$ & 0.169 \\
\hline N stage N1 vs. N0 & 2.982 & $1.798-4.945$ & $<0.001$ & 1.042 & $0.473-2.295$ & 0.918 \\
\hline M stage M1 vs. M0 & 2.947 & $1.054-8.243$ & 0.039 & 1.414 & $0.468-4.272$ & 0.539 \\
\hline TNM stage (III+IV) vs. (I+II) & 3.708 & $2.126-6.468$ & $<0.001$ & 3.695 & $1.357-10.060$ & 0.011 \\
\hline CRNDE high vs. low & 1.365 & $1.221-1.525$ & $<0.001$ & 1.309 & $1.160-1.478$ & $<0.001$ \\
\hline
\end{tabular}

HR, hazard ratio; CI, confidence intervals; IHCC, intrahepatic cholangiocarcinoma; TBIL, Total bilirubin; AFP, $\alpha$-fetoprotein; CEA, carcinoembryonic antigen; TNM, tumor-nodes-metastasis stages; CRNDE, colorectal neoplasia differentially expressed.

As for the colony formation assay, transfected HuCCT1 and HCCC9810 cells were seeded in $6 \mathrm{~cm}$ plates and then subjected to incubator for 7 days in an atmosphere of $5 \% \mathrm{CO}_{2}$ at $37^{\circ} \mathrm{C}$. Formed colonies were counted with light microscopy. All the experiments were performed in triplicate and repeated at least three times.

Cell migration and invasion assay. Cell migration ability was assessed by Transwell assay using $8 \mu \mathrm{m}$-pore-size chambers (BD, San Jose, CA, USA). Cells were seeded in the upper chamber, which was filled with $250 \mu \mathrm{l}$ serum-free medium. The lower chamber was filled with $600 \mu \mathrm{l}$ complete medium as chemoattractant. Then, the chambers were subjected to incubation at $37^{\circ} \mathrm{C}$ for $36 \mathrm{~h}$. Then, cells were fixed with $4 \%$ paraformaldehyde and stained with crystal violet. Finally, cells invaded to the lower surface of the filter were counted in five random fields using microscope. To measure the invasion ability of IHCC cells, Matrigel assay was conducted as the Transwell assay, except that each chamber was pre-coated with matrigel (BD, USA) before cell seeding. All the experiments were performed in triplicate and repeated at least three times.

Western blot analysis. Cells were harvested and rinsed with cold phosphate-buffered saline. Then, the total proteins were extracted with RIPA buffer (Thermo Scientific, Rockford, IL, USA) supplemented with protease inhibitors. After protein quantification, an equal amount of protein samples were size fractionated by SDS-PAGE, and separated proteins were electrotransferred onto PVDF membranes (Invitrogen, USA) in transfer buffer. Membranes were then incubated overnight at $4^{\circ} \mathrm{C}$ with primary antibody, followed by incubation with HRP-conjugated secondary antibody. Antibody-antigen complexes were detected by incubating with ECL detection reagent (Amersham Biosciences, Castle Hill, Australia). ImageJ software was utilized to determine the band intensity.

Statistical analysis. SPSS 17.0 software was utilized for statistical analyses (SPSS, Chicago, IL, USA). Data was presented as mean \pm standard deviation (Mean \pm SD). Statistically analysis was performed using the Student's t-test for continuous variables and Chi-square test for categorical variables. All tests were two-tailed. By utilizing the mean CRNDE expression level as the cutoff value, all involved patients were divided into the low CRNDE expression group and the high CRNDE expression group. Subquently, the Kaplan-Meier test was conducted to evaluate the survival data and the log-rank test was performed to compare the cumulative survival differencebetween the low CRNDE expression group and the high CRNDE expression group. The statistical significance level was set at 0.05 .

\section{Results}

LncRNA CRNDE is notably overexpressed in IHCC and significantly correlates with IHCC clinical progression. The expression of lncRNA CRNDE was evaluated in IHCC cell lines and clinical specimens. The result of RT-qPCR assay showed that CRNDE was remarkably up-regulated in IHCC cell lines compared with HIBEpics (Fig. 1A). Further 
investigation of CRNDE expression in 118 IHCC clinical specimens revealed that tumor tissues displayed much higher expression level relative to tumor adjacent tissues (Fig. 1B). Besides, the expression levels of CRNDE in IHCC patients with poor and undifferentiated grade, lymph node metastasis, tumor-nodes-metastasis (TNM) stage III and stage IV and tumor size no less than $5 \mathrm{~cm}$ were much higher than those with well and moderate grade, without lymph node metastasis, TNM stage I and stage II and tumor size less than $5 \mathrm{~cm}$, respectively (Fig. 1C-F).

Furthermore, with mean CRNDE expression level serving as the cutoff value, all IHCC patients were divided into the high CRNDE expression group $(\mathrm{n}=51)$ and the low CRNDE expression group $(\mathrm{n}=67)$. Statistical analysis between CRNDE expression and IHCC clinicopathological parameters found that high CRNDE expression was obviously correlated with poor and undifferentiated grade $(\mathrm{P}=0.046)$, lager tumor size $(\mathrm{P}=0.001)$, lymph node metastasis $(\mathrm{P}=0.001)$, and advanced TNM stage $(\mathrm{P}=0.024)$. (Table I) These findings suggested that CRNDE overexpression could promote the clinical progression of IHCC.

High CRNDE expression is an independent risk factor of IHCC poor prognosis. For further verifying the clinical significance of high CRNDE expression in IHCC, the prognostic value of CRNDE was evaluated. Intriguingly, high CRNDE expression was discovered to correlate with poorer overall survival (OS) rate $(\mathrm{P}<0.001)$, (Fig. $2 \mathrm{~A})$ and shorter progression-free survival (PFS) period $(\mathrm{P}<0.001)$ (Fig. 2B).

In addition, the risk factors of IHCC OS and PFS were statistically analyzed by univariate $\mathrm{COX}$ regression analysis and multivariate $\mathrm{COX}$ regression analysis. Univariate analysis revealed 5 risk factors for worse OS (Table II) and 6 risk factors for poor PFS (Table III) in IHCC patients, respectively. By further analyzing these factors with multivariate analysis, lymphovascular invasion $(\mathrm{HR}=0.530,95 \%$ $\mathrm{CI}=0.313-1.224, \mathrm{P}=0.018)$, advanced TNM stage $(\mathrm{HR}=3.695$, 95\% CI $=1.357-10.060, \mathrm{P}=0.011)$ and high $\mathrm{CRNDE}$ expression $(\mathrm{HR}=1.309,95 \% \mathrm{CI}=1.160-1.478, \mathrm{P}<0.001)$ were highlighted as independent risk factors of poor OS in IHCC. (Table II) Interestingly, these three factors, lymphovascular invasion ( $\mathrm{HR}=0.432,95 \% \mathrm{CI}=0.255-0.734, \mathrm{P}=0.002)$, advanced TNM stage $(\mathrm{HR}=2.484,95 \% \mathrm{CI}=1.104-5.586, \mathrm{P}=0.028)$ and high CRNDE expression (HR=1.305, 95\% CI=1.168-1.459, $\mathrm{P}<0.001$ ), were also identified as independent risk factors of shorter PFS period. (Table III).

Overexpression of IncRNA CRNDE could promote the proliferation of IHCC cells. To detect the functional role of CRNDE in IHCC cells, the expression of CRNDE was silenced with siRNAs in HuCCT1 cells (Fig. 2C), and ectopic overexpressed in HCCC9810 cells (Fig. 2D), respectively. Then, the effect of CRNDE on IHCC proliferation was evaluated with CCK-8 assay and colony formation assay. CRNDE silencing obviously inhibited the proliferation of HuCCT1 cells on CCK-8 assay and colony formation assay (Fig. 3A and 3B). Consistently, the proliferation ability of HCCC 9810 cells was notably increased with CRNDE ectopic overexpression (Fig. 3C and D). It was concluded that CRNDE could promote the proliferation of IHCC cells.
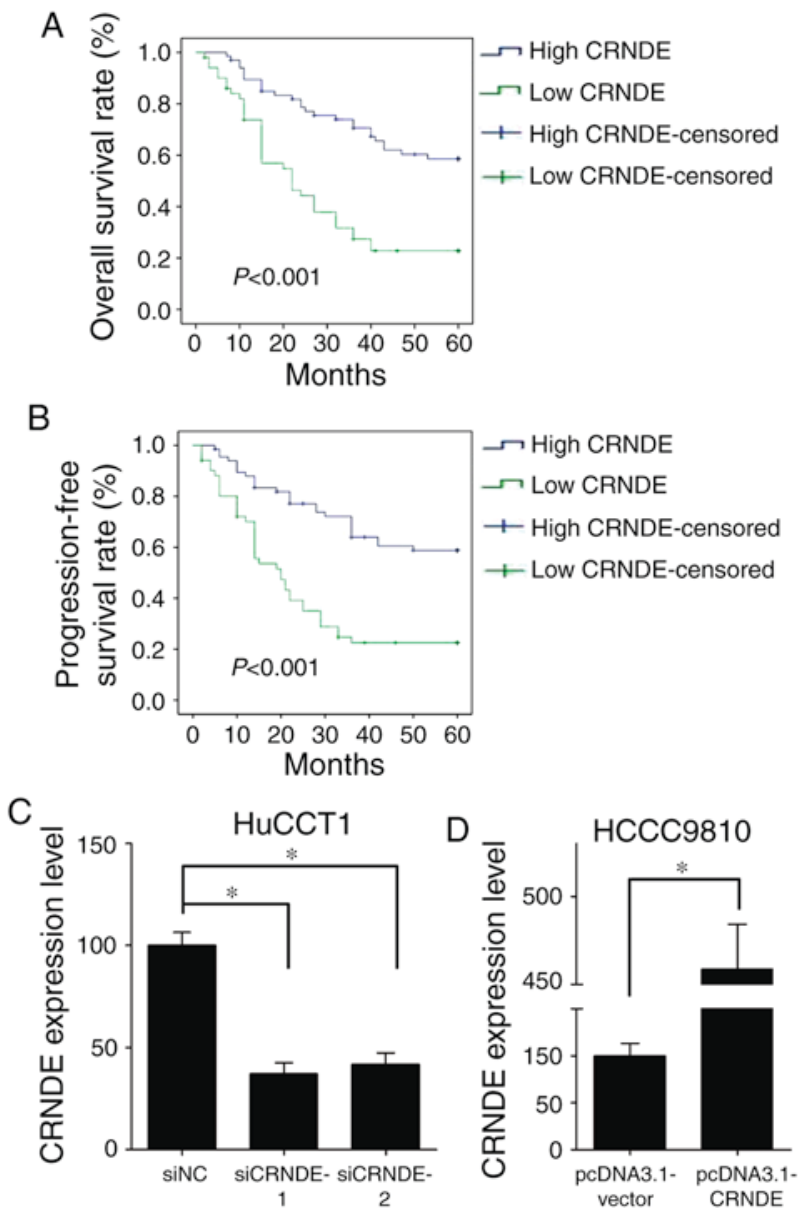

Figure 2. CRNDE overexpression predicts poor prognosis in IHCC. (A) High CRNDE expression in IHCC is associated with poor overall survival rate in IHCC. (B) High CRNDE expression in IHCC is associated with poor PFS rate in IHCC. (C) The expression of CRNDE was detected by RT-qPCR assay in HuCCT1 cells with CRNDE silencing using siCRNDE-1 and siCRNDE-2. (D) The expression of CRNDE was measured by RT-qPCR assay in HCCC9810 cells with CRNDE ectopic expression using pcDNA3.1-CRNDE. $\mathrm{P}<0.001$ in $\mathrm{A}$ and $\mathrm{B}$ based on log-rank test. ${ }^{*} \mathrm{P}<0.05$ in $\mathrm{C}$ and $\mathrm{D}$ based on Student's t-test. Data in C and D are represented as Mean \pm SD. CRNDE, colorectal neoplasia differentially expressed; IHCC, intrahepatic cholangiocarcinoma; RT-qPCR, reverse transcription-quantitative polymerase chain reaction; PFS, progression-free survival.

LncRNA CRNDE promotes IHCC metastasis by regulating $E M T$. Metastasis is the final stage of caner-caused death, the detailed mechanisms of tumor metastasis have been investigated for several decades but remain obscure at present. The present study confirmed CRNDE interference in HuCCT1 cells could repress the migration and invasion abilities on Transwell assay and Matrigel assay, respectively (Fig. 4A). While the motility of HCCC9810 cells was obviously increased with CRNDE up-regulation (Fig. 4B).

Epithelial-mesenchymal transition (EMT) is an essential process in the initiation and progression of cancer metastasis $(12,13)$. This study investigated the role of CRNDE on IHCC EMT by evaluating the expression of epithelial markers (E-cadherin and $\alpha$-catenin) and mesenchymal markers (N-cadherin and Vimentin) (14). As expected, the mRNAs levels of mesenchymal markers were notably reduced with CRNDE knock-down in HuCCT1 cells, while the mRNAs levels of epithelial markers were greatly 
Table III. Univariate and multivariate analysis of clinicopathologic features for progrerssion-free survival of IHCC patients.

\begin{tabular}{|c|c|c|c|c|c|c|}
\hline \multirow[b]{2}{*}{ Parameters } & \multicolumn{2}{|c|}{ Univariate analysis } & \multirow[b]{2}{*}{ P-value } & \multicolumn{2}{|c|}{ Multivariate analysis } & \multirow[b]{2}{*}{ P-value } \\
\hline & HR & $95 \% \mathrm{CI}$ & & HR & $95 \% \mathrm{CI}$ & \\
\hline Age $\geq 60$ years vs. $<60$ years & 1.152 & $0.704-1.885$ & 0.574 & & & \\
\hline Gender female vs. male & 0.676 & $0.388-1.180$ & 0.168 & & & \\
\hline TBIL $\geq 20.4 \mu \mathrm{mol} / 1$ vs. $<20.4 \mu \mathrm{mol} / 1$ & 1.227 & $0.736-2.045$ & 0.434 & & & \\
\hline $\mathrm{AFP} \geq 20 \mathrm{ng} / \mathrm{ml}$ vs. $<20 \mathrm{ng} / \mathrm{ml}$ & 1.605 & $0.972-2.648$ & 0.064 & & & \\
\hline $\mathrm{CEA} \geq 5 \mu \mathrm{g} / \mathrm{l} \mathrm{vs} .<5 \mu \mathrm{g} / \mathrm{l}$ & 1.274 & $0.776-2.093$ & 0.338 & & & \\
\hline CA19-9 $<100 \mathrm{U} / \mathrm{ml}$ vs. $\geq 100 \mathrm{U} / \mathrm{ml}$ & 0.810 & $0.496-1.323$ & 0.399 & & & \\
\hline Cirrhosis yes vs. no & 1.134 & $0.693-1.853$ & 0.617 & & & \\
\hline Satellitosis yes vs. no & 1.465 & $0.863-2.478$ & 0.158 & & & \\
\hline Perineural invasion yes vs. no & 1.206 & $0.727-2.002$ & 0.469 & & & \\
\hline Lymphovascular invasion no vs. yes & 0.430 & $0.262-0.704$ & 0.001 & 0.432 & $0.255-0.734$ & 0.002 \\
\hline Grade poor+undifferentiated vs. well+moderate & 1.679 & $1.107-2.770$ & 0.043 & 0.792 & $0.436-1.442$ & 0.446 \\
\hline Size $\geq 5 \mathrm{~cm}$ vs. $<5 \mathrm{~cm}$ & 1.474 & $0.898-2.418$ & 0.125 & & & \\
\hline T stage (T3+T4) vs. $(\mathrm{T} 1+\mathrm{T} 2)$ & 1.275 & $0.881-1.845$ & 0.198 & & & \\
\hline $\mathrm{N}$ stage N1 vs. N0 & 2.800 & $1.698-4.617$ & $<0.001$ & 1.294 & $0.646-2.590$ & 0.467 \\
\hline M stage M1 vs. M0 & 2.896 & $1.039-8.084$ & 0.042 & 1.311 & $0.437-3.928$ & 0.629 \\
\hline TNM stage (III+IV) vs. (I+II) & 3.438 & $1.994-5.928$ & $<0.001$ & 2.484 & $1.104-5.586$ & 0.028 \\
\hline CRNDE high vs. low & 1.366 & $1.224-1.525$ & $<0.001$ & 1.305 & $1.168-1.459$ & $<0.001$ \\
\hline
\end{tabular}

HR, hazard ratio; CI, confidence intervals; IHCC, intrahepatic cholangiocarcinoma; TBIL, Total bilirubin; AFP, $\alpha$-fetoprotein; CEA, carcinoembryonic antigen; TNM, tumor-nodes-metastasis stages; CRNDE, colorectal neoplasia differentially expressed.

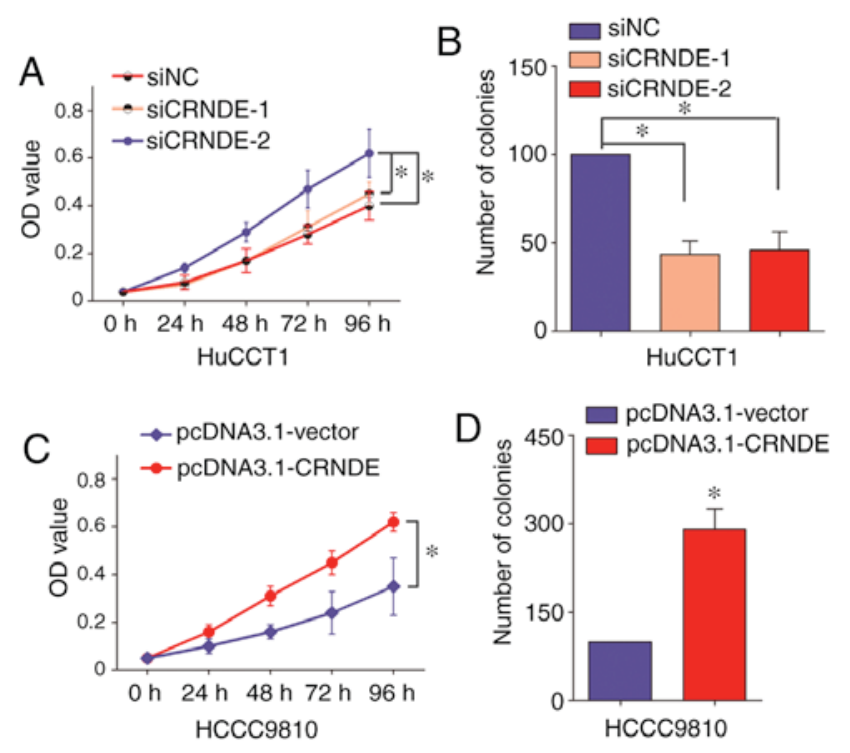

Figure 3. LncRNA CRNDE could promote the proliferation of IHCC cells. (A) CCK-8 assay was adopted to measure the proliferation ability of HuCCT1 cells with CRNDE deficiency. (B) The proliferation ability of HuCCT1 cells with CRNDE silencing was evaluated on colony formation assay. (C) Cell Counting kit- 8 assay was performed to measure the proliferation ability of HCCC 9810 cells with CRNDE up-regulation. " $\mathrm{P}<0.05$, as indicated. (D) The proliferation ability of HCCC 9810 cells with CRNDE overexpression was evaluated on colony formation assay. "P<0.05 vs. pcDNA3.1-vector, based on Student's t-test. Data are represented as Mean \pm SD. CRNDE, colorectal neoplasia differentially expressed; IHCC, intrahepatic cholangiocarcinoma.

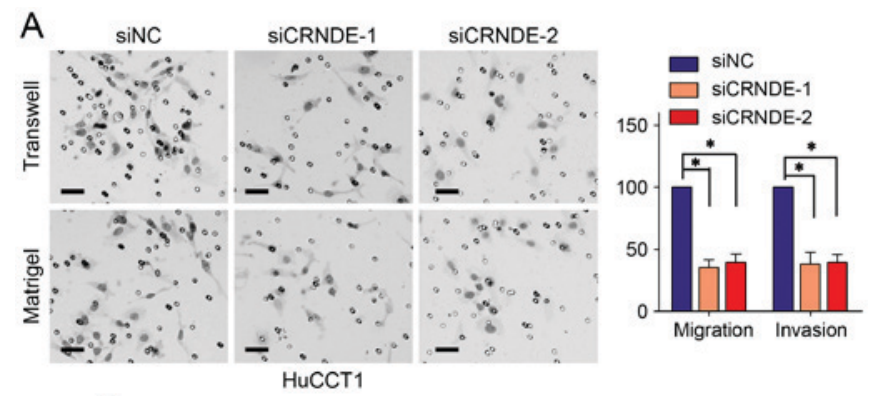

B pcDNA3.1-vector pcDNA3.1-CRNDE

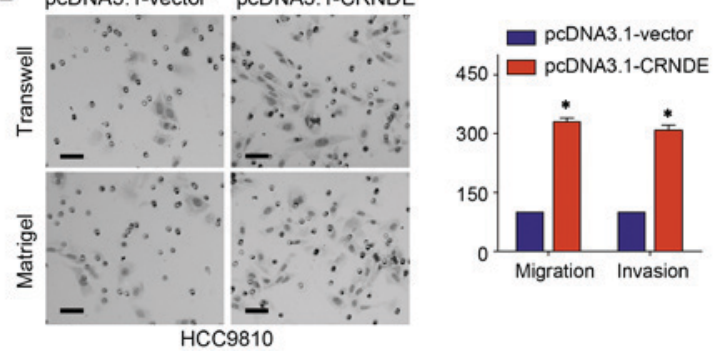

Figure 4. LncRNA CRNDE could increase the motility of IHCC cells (A) The migration and invasion abilities of HuCCT1 with CRNDE silencing were measured through Transwell assay and Matrigel assay. The left panel displays the typical figures. ${ }^{*} \mathrm{P}<0.05$, as indicated. (B) Transwell assay and Matrigel assays were performed to explore the migration and invasion of HCCC9810 cells with CRNDE overexpression. The left panel displays the typical figures. Scale bars, $50 \mu \mathrm{m} .{ }^{*} \mathrm{P}<0.05$ vs. pcDNA3.1-vector, based on Student's t-test. Data are represented as Mean \pm SD. CRNDE, colorectal neoplasia differentially expressed; IHCC, intrahepatic cholangiocarcinoma. improved with CRNDE silencing (Fig. 5A). Consistently, CRNDE overexpression in HCCC9810 cells increased the
mRNAs levels of mesenchymal markers, accompanied with down-regulation of epithelial markers (Fig. 5B). The 

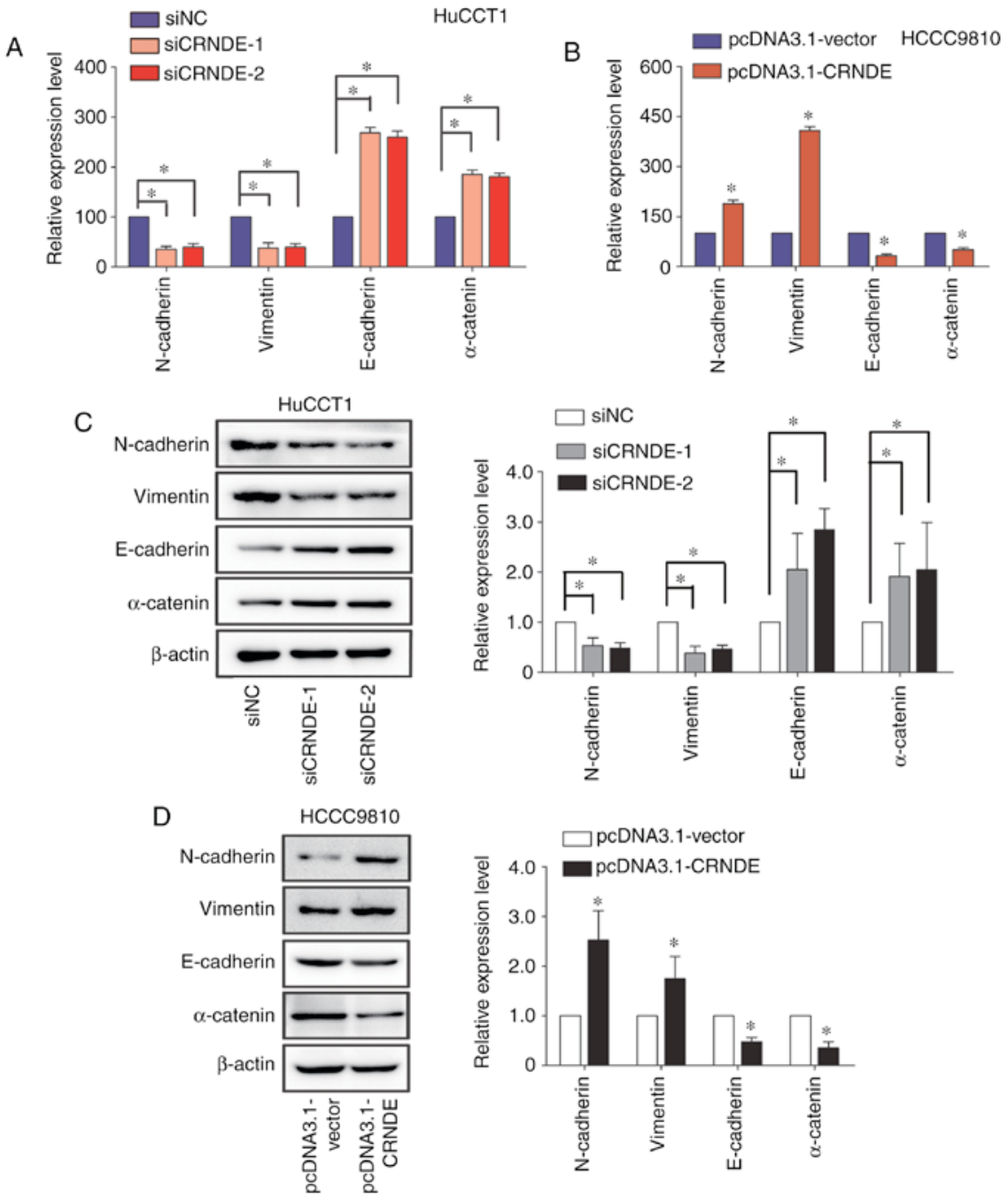

Figure 5. LncRNA CRNDE could facilitate the EMT of IHCC cells. (A) The mRNA levels of epithelial markers (E-cadherin and $\alpha$-catenin) and mesenchymal markers (Vimentin and N-cadherin) in HuCCT1 cells with CRNDE deficiency were investigated on RT-qPCR assay. ${ }^{*} \mathrm{P}<0.05$, as indicated. (B) The mRNA levels of epithelial markers (E-cadherin and $\alpha$-catenin) and mesenchymal markers (Vimentin and N-cadherin) in HCCC9810 cells with CRNDE up-regulation were investigated on RT-qPCR assay. ${ }^{*} \mathrm{P}<0.05$ vs. pcDNA3.1-vector. (C) Western blot was utilized to measure the protein levels of epithelial markers (E-cadherin and $\alpha$-catenin) and mesenchymal markers (Vimentin and N-cadherin) after CRNDE down-regulation in HuCCT1 cells. The bar charts of quantified western blots were shown in the right panel. "P<0.05, as indicated. (D) Western blot was utilized to measure the protein levels of epithelial markers (E-cadherin and $\alpha$-catenin) and mesenchymal markers (Vimentin and N-cadherin) after CRNDE overexpression in HCCC9810 cells. The bar charts of quantified western blots were shown in the right panel. "P $<0.05$ vs. pcDNA3.1-vector, based on Student's t-test. Data are represented as Mean \pm SD. CRNDE, colorectal neoplasia differentially expressed; EMT, epithelial-mesenchymal transition; IHCC, intrahepatic cholangiocarcinoma; RT-qPCR, reverse transcription-quantitative polymerase chain reaction.

results of the western blot assay further confirmed the findings of the RT-qPCR assay (Fig. 5C and D). Together, CRNDE could promote the metastasis of IHCC cells by facilitating EMT.

\section{Discussion}

Accumulating evidence has revealed that lncRNAs are essential participants in cancer biology. LncRNAs are involved in almost every aspect of cellular biology by acting as transcriptional modulators, splicing regulators, posttranscriptional processors, enhancers, molecular decoys for miRNAs, chromatin remodelers, or as guides for protein-protein, protein-DNA and protein-RNA interactions $(15,16)$. Recent literatures have indicated that IncRNAs are often expressed in a disease-, tissue- or developmental-specific manner, which makes a foundation for utilizing lncRNAs as biomarkers and therapeutic targets in different diseases. Moreover, the number of lncRNAs has recently been greatly expanded to triple the number of protein-coding genes (5). Therefore, lncRNAs are likely to serve as the basis for clinical diagnosis and treatment in oncology.

The present study discovered that lncRNA CRNDE was up-regulated in IHCC cells and tissues, and high CRNDE expression significantly promoted clinical progression of IHCC, including poorer differentiation, larger tumor size, lymph node metastasis, and advanced TNM stage. Further investigations found CRNDE up-regulation predicted poorer OS and earlier recurrence of IHCC patients. Moreover, CRNDE was revealed to be an independent risk factor of poor OS and PFS in IHCC. The results of in vivo studies verified that CRNDE could increase the proliferation ability 
of IHCC cells on CCK-8 assay and colony formation assay. By detecting the mRNA levels and protein levels of EMT markers, we confirmed CRNDE could facilitate the EMT of IHCC cells and thus promote the metastasis of IHCC cells, which was also confirmed by Transwell assay and Matrigel assay. All these results suggested that CRNDE could promote the progression of IHCC and may serve as a promising prognostic marker and therapeutic target of IHCC patients.

Increased CRNDE expression was first discovered by Graham and his colleagues (7) in the early stage of colorectal neoplasia, and was regarded as a promising tissue and plasma biomarker in colorectal cancer early detection. Since then, the functional roles and mechanisms of CRNDE in cancer development and progression have been gradually exhibited. It has been demonstrated that CRNDE was up-regulated in various solid tumors, including hepatocellular carcinoma, renal cancer, adrenocortical carcinoma, pancreatic cancer, prostate cancer, ovarian cancer, and leukemias (17). CRNDE is a multifunctional lncRNA and functions through different splice forms, which could provide specific functional scaffolds for regulatory complexes, such as the polycomb repressive complex 2 (PRC2) and CoREST chromatin-modifying complexes (18). Besides, CRNDE exhibited a very time- and tissue- specific pattern of expression. Recent literatures have discovered some other signaling pathways through which CRNDE exerts its functions. Zheng et al (19) demonstrated that CRNDE played an oncogenic role in human glioma stem cells through negatively regulating miR-186 expression. Besides, CRNDE was discovered to recruit the Deleted in Malignant Brain Tumors 1 (DMBT1) and Baculoviral IAP repeat Containing 1 (c-IAP1) through acting as a scaffold, which could accelerate gallbladder cancer development by promoting the PI3K-AKT pathway (20). In breast cancer, CRNDE was revealed to promote tumor growth through acting as a molecular sponge of miR-136, and thus hyperactivating the Wnt/ $\beta$-catenin signaling pathway (11). Therefore, various signaling pathways may underlie the functions and mechanisms of CRNDE in different cancers. The detailed mechanisms of CRNDE exerting its functions deserve further investigations.

In conclusion, the present study confirmed that CRNDE was up-regulated in IHCC cells and tissues. Statistical analyses found CRNDE expression was positively correlated with IHCC clinical progression, poor OS and PFS. Furthermore, univariate and multivariate COX regression analyses discovered that CRNDE overexpression was an independent risk factor of IHCC poor OS and PFS. The function of CRNDE was also confirmed in vitro assays by detecting its role in affecting the proliferation and metastasis of IHCC cells. Mechanistically, EMT was demonstrated to be accelerated with CRNDE up-regulation, which may be the underlying mechanism of CRNDE promoting IHCC metastasis. These findings indicated that CRNDE may be an essential prognostic marker and therapeutic target in IHCC.

\section{References}

1. Sirica AE, Dumur CI, Campbell DJ, Almenara JA, Ogunwobi OO and Dewitt JL: Intrahepatic cholangiocarcinoma progression: Prognostic factors and basic mechanisms. Clin Gastroenterol Hepatol 7 (11 Suppl): S68-S78, 2009.
2. Razumilava N and Gores GJ: Cholangiocarcinoma. Lancet 383: 2168-2179, 2014.

3. Aljiffry M, Abdulelah A, Walsh M, Peltekian K, Alwayn I and Molinari M: Evidence-based approach to cholangiocarcinoma: A systematic review of the current literature. J Am Coll Surg 208: 134-147, 2009.

4. Chen LL: Linking long noncoding RNA localization and function. Trends Biochem Sci 41: 761-772, 2016.

5. Evans JR, Feng FY and Chinnaiyan AM: The bright side of dark matter: lncRNAs in cancer. J Clin Invest 126: 2775-2782, 2016.

6. Adams BD, Parsons C, Walker L, Zhang WC and Slack FJ: Targeting noncoding RNAs in disease. J Clin Invest 127: 761-771, 2017.

7. Graham LD, Pedersen SK, Brown GS, Ho T, Kassir Z, Moynihan AT, Vizgoft EK, Dunne R, Pimlott L, Young GP, et al: Colorectal neoplasia differentially expressed (CRNDE), a novel gene with elevated expression in colorectal adenomas and adenocarcinomas. Genes Cancer 2: 829-840, 2011.

8. Gao H, Song X, Kang T, Yan B, Feng L, Gao L, Ai L, Liu X, $\mathrm{Yu} \mathrm{J}$ and $\mathrm{Li} \mathrm{H}$ : Long noncoding RNA CRNDE functions as a competing endogenous RNA to promote metastasis and oxaliplatin resistance by sponging miR-136 in colorectal cancer. Onco Targets Ther 10: 205-216, 2017.

9. Yu B, Ye X, Du Q,Zhu B, Zhai Q and Li XX: The long non-coding RNA CRNDE promotes colorectal carcinoma progression by competitively binding miR-217 with TCF7L2 and enhancing the Wnt $/ \beta$-catenin signaling pathway. Cell Physiol Biochem 41: 2489-2502, 2017.

10. Shao K, Shi T, Yang Y, Wang X, Xu D and Zhou P: Highly expressed lncRNA CRNDE promotes cell proliferation through Wnt $/ \beta$-catenin signaling in renal cell carcinoma. Tumour Biol: Oct 6, 2016 (Epub ahead of print).

11. Huan J, Xing L, Lin Q, Xui H and Qin X: Long noncoding RNA CRNDE activates Wnt/ $\beta$-catenin signaling pathway through acting as a molecular sponge of microRNA-136 in human breast cancer. Am J Transl Res 9: 1977-1989, 2017.

12. Tsai JH and Yang J: Epithelial-mesenchymal plasticity in carcinoma metastasis. Genes Dev 27: 2192-2206, 2013.

13. De Craene B and Berx G: Regulatory networks defining EMT during cancer initiation and progression. Nat Rev Cancer 13: 97-110, 2013.

14. Zeisberg $M$ and Neilson EG: Biomarkers for epithelial-mesenchymal transitions. J Clin Invest 119: 1429-1437, 2009.

15. Bartonicek N, Maag JL and Dinger ME: Long noncoding RNAs in cancer: Mechanisms of action and technological advancements. Mol Cancer 15: 43, 2016.

16. Chandra Gupta S and Nandan Tripathi Y: Potential of long non-coding RNAs in cancer patients: From biomarkers to therapeutic targets. Int J Cancer 140: 1955-1967, 2017.

17. Ellis BC,Molloy PL and Graham LD: CRNDE: A long non-coding RNA involved in canceR, neurobiology, and DEvelopment. Front Genet 3: 270, 2012.

18. Khalil AM, Guttman M, Huarte M, Garber M, Raj A, Rivea Morales D, Thomas K, Presser A, Bernstein BE, van Oudenaarden A, et al: Many human large intergenic noncoding RNAs associate with chromatin-modifying complexes and affect gene expression. Proc Natl Acad Sci USA 106: 11667-11672, 2009.

19. Zheng J, Li XD, Wang P, Liu XB, Xue YX, Hu Y, Li Z, Li ZQ, Wang ZH and Liu YH: CRNDE affects the malignant biological characteristics of human glioma stem cells by negatively regulating miR-186. Oncotarget 6: 25339-25355, 2015.

20. Shen S, Liu H, Wang Y, Wang J, Ni X, Ai Z, Pan H, Liu H and Shao Y: Long non-coding RNA CRNDE promotes gallbladder carcinoma carcinogenesis and as a scaffold of DMBT1 and C-IAP1 complexes to activating PI3K-AKT pathway. Oncotarget 7: 72833-72844, 2016.

This work is licensed under a Creative Commons Attribution-NonCommercial-NoDerivatives 4.0 International (CC BY-NC-ND 4.0) License. 